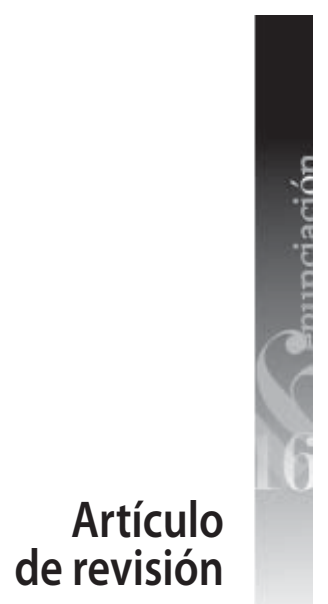

\title{
La enseñanza reflexiva y progresiva de la lengua oral
}

\section{Progressive and reflective teaching of oral language}

Yolima Gutiérrez Ríos

Universidad Distrital Francisco José de Caldas*
Recibido: 12 de noviembre de 2010

Revisado: 10 de diciembre de 2010 Aprobado: 24 de enero de 2011

\section{Resumen}

Este artículo ${ }^{1}$ presenta los resultados finales de la tesis doctoral realizada por la autora, cuya problemática se sitúa en la ausencia de enseñanza reflexiva y progresiva sobre la lengua oral en el contexto de la educación media colombiana. En particular, se exponen de manera resumida algunos referentes teóricos y metodológicos y se ahonda en el significado otorgado a la oralidad, una de las ocho categorías disciplinares y didácticas identificadas. El estudio interpretativo se centra en el pensamiento del profesor de castellano como lengua materna y, particularmente, analiza su "conocimiento didáctico del contenido lingüístico-discursivo oral", con fundamento en sus concepciones disciplinares y didácticas acerca de la enseñanza de la lengua oral. Aporta conocimientos sobre cómo concibe el profesor la enseñanza de la lengua oral, qué sabe y qué enseña (conocimiento disciplinar), cómo hace enseñable un contenido específico (conocimiento didáctico), en síntesis, qué actividades de enseñanza planea, desarrolla y evalúa y, en general, cómo operan los principios y estrategias de manejo y gestión de la clase que trascienden el ámbito de la materia (conocimiento pedagógico). Esta caracterización e interpretación de concepciones derivadas del discurso y la acción didáctica del profesorado son los principales aportes de la investigación y sustentan la elaboración de lineamientos para la formación y actualización docente, encaminados al desarrollo de la competencia discursiva oral, especialmente en la educación media.

Palabras clave: Oralidad, didáctica, lengua materna, concepciones, conocimiento profesional del profesor, educación media.

* Doctora en Educación de la Universidad Distrital Francisco José de Caldas. Profesora de Lengua Castellana y Literatura de la Escuela Normal Superior Distrital María Montessori. Profesora hora cátedra de la Maestría en Pedagogía de la 1 Lengua Materna de la Universidad Distrital Francisco José de Caldas. Correo: yolimagr@yahoo.es

1 Artículo fruto de la tesis doctoral “Análisis de las concepciones disciplinares y didácticas sobre la lengua oral en la escuela colombiana actual: un estudio sobre el desarrollo de la competencia discursiva oral en Educación Media”. 


\section{Abstract}

This article presents the final outcomes from a doctoral thesis, carried out by the author, which states as a problem the lack of a progressive and reflective teaching of the oral language in the context of tenth and eleventh grades of Secondary Schools in Colombia. Particularly, it says in a succinct way some theories and methodologies in the field, and deepen the meaning that has been given to orality, which is one of the eight disciplinary and didactic identified categories in this research. The Spanish as a mother tongue teacher's (SMTT hereafter) thought is at the core of the interpretive study. Especially, it analyzes the "teacher's didactic knowledge of the oral linguistic-discursive content", based on their disciplinary and didactic conceptions of oral language teaching. The study also contributes to enrich the knowledge on how the SMTT conceives the oral language teaching, what he knows and teaches (disciplinary knowledge), and how he makes teachable a specific content (didactic knowledge). In summary, What teaching activities does he plans, develop, and assess?, and generally speaking, How do principles and classroom management strategies that transcend the subject operate? This description and interpretation of conceptions derived from Spanish staff's discourse and didactic actions is viewed as the main contribution of the research study. In addition, it supports the guidelines for teachers' training and updating towards the development of oral discursive competence, especially in tenth and eleventh grades of High School.

Key words: Orality, didactic, mother tongue, conceptions, teacher's professional knowledge, tenth and eleventh grade education.

\section{La oralidad en el contexto sociocultural actual}

El macrocontexto de esta investigación es el escenario social contemporáneo, que revela una serie de fe- nómenos históricos y culturales complejos y contradictorios. En las últimas décadas, los ámbitos de la vida pública y privada se han visto inmersos en continuos cambios, cifrados en el fenómeno de la globalización y el avance del capitalismo posindustrial, la difusión del modelo democrático como forma ideal de organización de la polis, los incontables avances tecnológicos y comunicativos que constituyen la sociedad de la información. En materia educativa se realizan esfuerzos considerables para reducir el alto índice de analfabetismo y formar al ciudadano desde una perspectiva de eficacia y, en general, se observan avances importantes de carácter científico, cultural, social, económico y político.

Estudiar el lenguaje en este escenario implica reconocer su adaptación a la vertiginosidad de estos cambios históricos y sociales. Además, reconocer su papel como instrumento de intelección, acción y poder en las prácticas sociales, que en realidad lo son de producción de discursos en situaciones de interacción. En este sentido, se comparte con Bourdieu (2001) que: "las relaciones de comunicación son intercambios lingüísticos y sobre todo son relaciones de poder simbólico donde se actualizan las relaciones de fuerza entre los locutores y sus respectivos grupos" (p. 11). En esta estructura se naturaliza el papel dominante del lenguaje, y se legitima y reproduce el orden social y la distribución desigual del capital cultural (Bourdieu, 2001; Foucault, 1973, 1978; Lyotard, 2004).

En efecto, el sujeto posmoderno transita entre el poder y la riqueza del lenguaje, y se torna frágil e indefenso cuando no cuenta con cierto desarrollo de su competencia discursiva, con lo que queda expuesto a la marginación y exclusión, en razón a que su propio capital verbal y comunicativo no tiene valor en los mercados oficiales (escolar, político, administrativo, etc.) o en los espacios donde esté comprometida su producción verbal (Bourdieu, 2001). Esta es una situación lamentablemente recurrente en países como 
Colombia, donde la mayoría de la población espera que otros hablen por ella y lleven su voz ante quienes ejercen el poder político o judicial, antes que usar su derecho a expresarse y a ser escuchados, según lo confieren la Constitución y la ley. Así, se instituye la voz del más fuerte como portadora de la realidad de los hechos y se desconoce la de quien, a pesar de haber sido víctima de la problemática de su entorno, no tiene la competencia oral para describirla y aportar soluciones.

Esta investigación comparte los anteriores presupuestos y centra la mirada en el papel del lenguaje como instrumento de creación, construcción de saber e interacción social; es decir, se considera que los cambios actuales generan nuevas exigencias de diálogo entre las culturas y entre los sujetos y su realidad, condiciones fundamentales para el desarrollo de medios de reinvención, democratización y revolución (Bronckart, 1985, 2004; Brünner, 1998; De Certeau, 1979; Freire, 1969; Martínez, 1991; Rodríguez, 2000). De ahí que la creación sea considerada la actividad vital y emancipadora del hombre contemporáneo; una capacidad que lo fortalece frente al juego de fuerzas en tensión entre la ciencia, la tecnología y la sociedad (Lyotard, 2004). Es en esta complejidad de relaciones donde el lenguaje ratifica su papel como mediador en la representación que los sujetos hacen de su entorno, en las interrelaciones con el otro y en la estructuración de las operaciones de razonamiento, decisivas en los procesos y actividades propias de la construcción de conocimiento. Esta actividad lingüística compromete ineludiblemente a la oralidad como un acto comunicativo de carácter humano, presente en las distintas épocas y contextos sociohistóricos.

No obstante, al estudiar en este macrocontexto la oralidad como actividad de hablar y escuchar y su incidencia en la formación del sujeto, en la construcción del conocimiento y en la interacción so- cial, se identifica a la vez una variedad de tensiones, vacíos y aperturas que configuran una brecha profunda entre las formas como se concibe la oralidad en los discursos que circulan en el territorio escolar y social y los desarrollos recientes de distintos campos disciplinares. Para representar esta brecha se acude a la metáfora de lo instituido e instituyente de Castoriadis (1997), dos dimensiones que ponen en escena la problemática de esta investigación. La dimensión instituida de la oralidad da cuenta de situaciones que permanecen y tienden a perpetuarse y la dimensión instituyente de la oralidad es portadora de innovación, cambio y renovación. El estudio de estas dos dimensiones da como resultado que:

1. La oralidad adquiere el estatuto de campo interdisciplinar, en cuanto se reconoce un conjunto de condiciones ontológicas, gnoseológicas, axiológicas, antropológicas, lingüísticas, discursivas, pedagógicas y didácticas que posibilitan su valoración como objeto de estudio y su comprensión como objeto de enseñanza y aprendizaje en la sociedad y la escuela de hoy.

2. La oralidad, entendida como la actividad de hablar y escuchar, adquiere un sentido reflexivo por su capacidad de sensación, de contacto, de fascinación, de reconocer cierto sabor, olor y fluidez de la palabra, que hacen que ella despierte una mayor actividad sensorial y sea más sensual que la escritura. Esta vitalidad de la oralidad determinada por una necesaria copresencialidad lleva a pensar que es difícil concebirla solo como el acto de hablar (connatural al hombre) o referirse solo al hablante, ya que más bien se trata de un interlocutor semejante a un actor o actriz, por cuanto la acción implícita en su discurso rebasa los límites del discurso oral y pone otros discursos en acción (Alcántara). Este carácter performativo de la oralidad da cuenta del alcance semántico de los gestos, posturas y 
actitudes, como signos culturales de valor simbólico² (Poyatos).

3. En este orden de ideas, la oralidad no puede entenderse como la simple enunciación, sino como la acción de enunciar, lo cual supone una actividad portadora de un discurso permeado por elementos sociohistóricos, culturales, políticos, semióticos, etc. Es decir, emergen voces de otros $^{3}$ que se enriquecen con los recursos propios de la oralidad $\mathrm{d}^{4} \mathrm{y}$ hacen posible su carácter multicanal; por ello las personas pertenecientes a determinados grupos sociales logran establecer una comunicación oral efectiva e incluso intercultural. Así, pues, una condición sine qua non de las actividades de hablar y escuchar en la Modernidad es su determinación de lo humano mediante el contacto, la presunción de cercanía y la acción ${ }^{5}$.

\section{El conocimiento profesional de los profesores}

A tenor de las tensiones, vacíos y aperturas presentadas en el apartado anterior, se estudian las concepciones de los profesores del castellano como lengua materna sobre la enseñanza de la lengua oral, en el marco del programa de investigación del "conocimiento profesional del profesor". Se trata, entonces, de analizar cómo concibe este la enseñanza de la lengua oral, qué sabe y qué enseña (conocimiento disciplinar), cómo hace enseñable un contenido específico (conocimiento didáctico); es decir, qué actividades de enseñanza privilegia, desarrolla y evalúa y, en general, cómo operan los principios y estrategias generales de manejo y gestión de la clase que trascienden el ámbito de la materia (conocimiento pedagógico). Esto es, estudiar el pensamiento del profesor y particularmente su conocimiento didáctico del contenido lingüísticodiscursivo oral.

El volumen de investigaciones derivadas del paradigma del "pensamiento del profesor" -o "pensamientos del profesor", como los denomina Marcelo (1987), o de los "procesos de pensamiento de los docentes", como los describen Clark y Peterson (1990), o del "paradigma mediacional cognitivo", según Winne y Marx (1977), o del conocimiento profesional del profesor, desde la perspectiva de Shulman (1986a, 2001)- muestra por lo menos dos tendencias claras. Por una parte, se observa un énfasis en la generación de modelos preestablecidos que le indican al profesor cómo ser racional en su actuación ${ }^{6}$. Por otra parte, se advierte una transición de esta tendencia cognitivista a otra alternativa más contextualista, en tanto se reconoce el mundo particular, complejo y cambiante del aula que exige diversos modos de actuación por parte del profesor y la capacidad de este para crear, investigar, adecuar o elaborar sus propios esquemas de intervención.

2 Además, da cuenta del carácter complementario de la teatralidad (performatividad de la oralidad) y la oralidad.

3 El hablante, según Bajtín (1982), no acude al diccionario cuando habla, sino que busca las palabras en la boca de otras personas, cuando estas se produjeron en otros contextos y con otras intenciones.

4 Por ejemplo, ciertos recursos fonéticos están presentes durante los intercambios verbales orales (paralingüística), también, actitudes, posturas, hábitos culturales que constituyen signos que pueden variar de un grupo social a otro (proxémica) y el caudal comunicativo a cargo de los gestos y movimientos corporales de percepción visual, auditiva o tangible (cinésica).

5 Esta naturaleza actuativa e interactiva de la oralidad se sustenta en la perspectiva bajtiniana que propone entender el uso de la lengua como un diálogo vivo y no como un código.

6 Propio de la psicología cognitivista. 
De este modo, se focalizan los problemas del contenido del pensamiento del profesor $^{7}$, esto es, se explora la naturaleza del conocimiento de los profesores, sus características, su forma y contenido (Grossman, Wilson y Shulman), a partir de la articulación entre el razonamiento pedagógico y la acción situada (Shulman, 1986b), lo cual señala que se centra la atención en aspectos socioantropológicos y filosóficos de la teoría social y la teoría crítica de la enseñanza (Marcelo, 1987; Perafán, 2004; Perafán y Adúriz-Bravo, 2005; Shulman, 1986a). Esta transición ${ }^{8}$ la describe Marcelo en los siguientes términos:

La investigación sobre el pensamiento del profesor teacher thinking (Marcelo, 1987), predominante en la década de los años 80 dio paso a una gran preocupación por el conocimiento del profesor teacher knowledge. El cambio de interés vino motivado por el descubrimiento de la estrechez del análisis psicológico y cognitivo de la investigación, así como de una creciente influencia contextual y epistemológica que reconocía, gracias a la aportación, entre otros, de Donald Schön $(1982,1987)$, el hecho evidente de que los docentes son profesionales que desarrollan una epistemología de la práctica, es decir, que generan conocimiento sobre la enseñanza que merece la pena ser investigado.
De este modo, se reconoce el pensamiento práctico, el pensamiento orientado a la acción, el cual emerge y se transforma en la reflexión sobre la práctica (Schön). En este marco los estudios sobre el conocimiento profesional del profesor se orientan a profundizar en los diferentes componentes del conocimiento del profesor, entre otros: "conocimiento profesionalizado del contenido", "conocimiento psicopedagógico general", "conocimiento del contexto escolar" y "conocimiento didáctico del contenido" (Shulman). Así, pues, se centra la atención en lo que un profesor debe saber y saber hacer para enseñar una determinada materia. Por ello, Porlán y Rivero (1998) plantean la existencia de un "conocimiento profesional dominante" (con un bajo nivel de integración entre el saber académico, las teorías implícitas, las rutinas y guiones, y los principios y creencias), el cual es de carácter mayoritario, y un "conocimiento profesional deseable" (con un alto nivel de integración de los componentes mencionados) como una tendencia posible.

El saber o el conocimiento del profesor ${ }^{10}$ se representan como una forma de discurso que señala un nuevo derrotero en las investigaciones, al articular el estudio de creencias, concepciones y modalida-

$7 \quad$ Por ejemplo, las dimensiones relacionadas con qué y por qué piensa y conoce, de tal forma que se pueda establecer un flujo de relaciones y una visión más global del profesor y su proceso de enseñanza.

8 Cabe aclarar que la aparición de nuevas categorías de análisis y del mismo programa del conocimiento profesional del profesor no significa la desaparición del anterior (pensamiento del profesor), sino la suma de aportes a dos tendencias.

9 El conocimiento didáctico del contenido (CDC o pedagogical content knowledge) es un componente fundamental en la formación y en la actualización profesional del profesorado. Se orienta a describir la manera como los profesores comprenden la materia y proceden a transformarla didácticamente, reconociendo también los demás tipos de conocimiento involucrados, es decir, el CDC se relaciona con las formas de representar la materia o de hacer "enseñables" y "aprendibles" tópicos particulares de la misma.

10 Cabe aclarar que en esta investigación se asumen indistintamente los conceptos de conocimiento y saber, a pesar de las diferencias que se establecen entre estos, cuando se le asignan al primero aquellos saberes que el profesor moviliza durante su acción didáctica (gestión de la clase y de la materia) y que provienen de operaciones intelectuales complejas, y al segundo, un sentido más amplio que implica un conjunto de conocimientos sustentados en el acto de enseñar en un contexto particular (formación inicial y continua, currículo y socialización escolar, conocimiento de la disciplina, conocimiento experiencial), los cuales se traducen en una actitud, una disposición y una práctica. 
des del conocimiento en condiciones contextuales determinadas. De ahí que en la década del noventa y la primera del nuevo milenio aparezcan en la escena investigativa autores que realizan aportes importantes al estudio de las concepciones y de otras construcciones mentales del profesor ${ }^{11}$ como una de las áreas de investigación más prometedoras en la actualidad (García y Cañal, 1995; Porlán y Rivero, 1998; Thompson, 1992; Valbuena, 2007, entre otros).

\section{Las concepciones como sistema de ideas en la línea del conocimiento profesional del profesor}

Con esta visión del conocimiento profesional, en las últimas décadas el Proyecto Curricular de Investigación y Renovación Escolar (IRES) ${ }^{12}$ ha realizado importantes desarrollos teóricos y metodológicos en el estudio de las concepciones y los obstáculos epistemológicos de los profesores. Particularmente, ha centrado la atención en identificar y caracterizar las concepciones de los profesores sobre la ciencia, la enseñanza y el aprendizaje y en establecer hipótesis acerca del conocimiento profesional deseable y de la posible progresión de este conocimiento (Porlán, 1987, entre otros). Por tanto, se comparte que si bien las concepciones son estables en la memoria semántica a largo plazo, también pueden ser activadas y actualizadas. Este carácter evolutivo de las concepciones de los profesores implica un componente epistemológico, en razón a que dicha evolución exige un proceso de integración y transformación de saberes orientados a formular un de- terminado conocimiento escolar y a comprender los procesos que facilitan su construcción. De ahí que considere las concepciones como:

\begin{abstract}
Un conjunto ideas y formas de actuar que tienen los profesores, que guardan relación más o menos directa con el conocimiento escolar y con sus procesos de construcción y facilitación, sean estas ideas de un nivel más epistemológico, didácticocurricular, metodológico, experiencial, etc., o se manifiesten de forma más o menos tácita o explícita (Porlán et ál., 1996, p. 161).
\end{abstract}

Por tanto, una parte importante de las concepciones y de las actuaciones de los profesores reflejan una determinada visión epistemológica y esta visión juega un papel estructurador, bloqueando o dinamizando, fragmentando o integrando parcelas importantes de su conocimiento profesional. Se comparte esta noción de las concepciones como sistema de ideas, como un tipo de conocimiento del profesor que él construye en sus interacciones con la sociedad y la cultura y que es susceptible de actualización. Esta proyección hacia el conocimiento profesional deseable no es para Porlán et ál. (1996) una visión idealista, sino una posibilidad cambiante, representada mediante una hipótesis de progresión o de referencia en cada nivel, en la perspectiva de reconocer las posibles evoluciones de las concepciones de los profesores:

- Nivel inicial (N1): en el que predominan concepciones propias de una enseñanza y aprendizaje academicista y transmisionista que dan cuenta de un modelo didáctico tradicional (suele ser de tendencia mayoritaria).

11 Tales como: creencias, valores, juicios, axiomas, opiniones, ideologías, percepciones, sistema conceptual, preconcepciones, disposiciones, teorías implícitas, teorías explícitas, teorías personales, procesos mentales internos, reglas de la práctica, principios prácticos, etc., orientadas a comprender su naturaleza y las posibles relaciones con la acción social o pedagógica, aunque su entramado teórico y sus tenues fronteras estén aún en proceso de construcción.

12 Liderado por el grupo español de Didáctica e Investigación Escolar (DIE), cuya producción investigativa se sitúa en la línea del conocimiento profesional del profesor. 
- Nivel intermedio (N2): correspondiente a planteamientos o alternativas de innovación que intentan superar el modelo tradicional de la enseñanza y aprendizaje (propone planteamientos diferentes para superar la tendencia mayoritaria).

- Nivel de referencia (N3): trata de superar las dificultades de los niveles anteriores y se aproxima al conocimiento profesional deseable (para los fines del presente estudio, se aproxima al conocimiento lingüístico-discursivo oral deseable desde una perspectiva colectiva e investigativa).

No se trata de una visión idealista de la enseñanza, sino de una posibilidad cambiante, representada en hipótesis de progresión o de referencia en cada nivel que sustentan la perspectiva de actualizar las concepciones disciplinares y didácticas de los profesores. Se trata, entonces, de una proyección hacia el conocimiento profesional deseable (Porlán et ál., 1996). Para ello es importante entender dicha evolución desde la propuesta de Porlán, Rivero García y Martín, quienes plantean entenderlas desde tres perspectivas: 1 . la constructivista, para la cual las concepciones son al mismo tiempo "herramientas" para interpretar la realidad y conducirse en ella y "barreras" que impiden adoptar perspectivas y cursos de acción diferentes (noción construida por los autores basados en los aportes de Bachelard; Claxton; Novak; Pope y Gilbert); 2. la sistémica y compleja, en la cual las concepciones se consideran sistemas de ideas en evolución (según aportes de García; Morín); y 3. la perspectiva crítica, que implica la visión integradora de las relaciones entre conocimiento, ideología y cotidianidad (los autores reconocen el aporte de Apple; Habermas). Por tanto, el acercamiento a este proceso de comprensión de la acción docente a través de sus concepciones posibilita encontrar otros caminos para fortalecer la enseñanza de lengua oral.

\section{Las concepciones disciplinares y didácticas presentes en la enseñanza de la lengua castellana como lengua materna en Colombia}

Las teorías lingüísticas contemporáneas plantean postulados filosóficos, epistemológicos y pedagógicos sobre el lenguaje y la lengua de los cuales se derivan instrumentos conceptuales de base, que a su vez constituyen nuevas epistemes denominadas "saberes de referencia" o "saberes disciplinares". Su transposición o transformación al campo educativo, a través de orientaciones curriculares y propuestas de renovación de las prácticas de enseñanza de la lengua materna, a juicio de diferentes teóricos y pedagogos de la lengua, nacionales y extranjeros (Bronckart, 1985; Camps, 1993; Rey, Borja, Jaimes y Rodríguez, en prensa, entre otros), ha generado múltiples tensiones en las relaciones que se establecen entre los saberes disciplinares relativos a la lengua materna, los saberes a enseñar y los saberes enseñados.

En esta investigación se consideran cuatro tendencias dominantes en la enseñanza lingüística: la tradicional (centrada en aspectos normativos de la lengua); la estructuralista (sustentada en la estructura y función de la lengua); la generativa transformacional (fundamentada en principios generadores que posee el hablante-oyente) y la comunicativa y contextualista (que enfatiza aspectos comunicativos, discursivos y socioculturales de la lengua).

Los avances de las ciencias del lenguaje en las últimas tres décadas junto con los cambios socioculturales han sido decisivos en la refundación de la enseñanza de la lengua castellana como lengua materna. Así, se evidencia un interés por establecer un diálogo entre las teorías del lenguaje y los objetivos de la enseñanza de la lengua castellana en la escuela, con la perspectiva de asumir la lengua como una herramienta para representar el mundo e interactuar en 
él. En esta evolución de las ciencias del lenguaje y la complejidad de su coexistencia y transformación didáctica, Camps (1993) identifica tres tendencias en el momento actual: una orientada a incidir en el desarrollo mental y social del individuo (Halliday, 1989); otra orientada por los estudios de la psicología del desarrollo y el análisis del discurso (Bajtín, 1982; Dijk, 2000; Vigotsky, 1991), centradas en las interacciones entre los agentes educativos y en el análisis de las producciones orales y escritas, y una tercera orientación que articula las dos líneas anteriores y toma como objeto de investigación el contexto, como sistema de actividades desde distintos campos disciplinares (Bronckart, 2004).

Es así como el interés por el estudio de la didáctica de las lenguas ha ido aumentado debido a la necesidad de comprender la complejidad del objeto de investigación, reflejada en la diversidad de disciplinas implicadas (análisis del discurso, sociología, antropología, etc.) y de contextos socioculturales y escolares. En concreto, se busca otorgar un estatuto propio a la enseñanza y el aprendizaje de las lenguas que "libere" de la dependencia de otros campos disciplinares, sin que ello signifique que no se siga nutriendo de sus aportes y saberes, en el sentido dado por Milian y Camps, quienes explican la configuración de la didáctica como un espacio de interrelación de diversos campos donde se transforma dicha confluencia de saberes. Sin duda no se están refiriendo a la suma de aportes teóricos de estos campos, sino a considerar la didáctica de las lenguas como un espacio de reflexión e investigación (Camps; Litwin, entre otros).

\section{Diseño metodológico de la investigación}

En relación con la perspectiva metodológica, la investigación es de carácter cualitativo con enfoque interpretativo o hermenéutico. El acercamiento al objeto de estudio se realiza mediante un diseño de investigación etnográfico tridimensional que com- promete procesos simultáneos de caracterización (descripción, descomposición y desagregación de componentes relativos al fenómeno que se estudia), interpretación (explicación de posibles relaciones entre categorías, conceptos o variables encontradas) y reconstrucción del sentido (mirada crítica y ampliada de las categorías implicadas en el análisis), con el propósito de inducir hallazgos susceptibles de conceptualizar.

Se opta por la combinación de dos métodos: el análisis sistemático del contenido (que consiste en formular inferencias sobre datos verbales y no verbales y fenómenos simbólicos) y el análisis clínico de la acción didáctica (se centra en el discurso didáctico como constructor de la acción didáctica del profesor), con el fin de contar con elementos suficientes para abordar la compleja tarea de develar las concepciones didácticas y disciplinares de los profesores sobre la enseñanza de la lengua oral y establecer sus implicaciones en la acción didáctica.

Los datos empíricos están constituidos por entrevistas, cuya información se confirma mediante la técnica de grupos de discusión y secuencias didácticas validadas a través de la autoconfrontación conjunta. La triangulación y análisis de la información se realiza mediante la comparación constante, primero, en el nivel del discurso, donde se correlacionan las entrevistas con los grupos de discusión y algunos momentos de la planeación de las secuencias de enseñanza; segundo, en el nivel de la acción didáctica, donde se correlacionan episodios de la secuencia didáctica con la autoconfrontación conjunta. De este modo se llega a la interpretación de la complejidad de las relaciones entre las concepciones disciplinares y didácticas presentes en el discurso y la práctica pedagógica del maestro, para establecer su incidencia en una enseñanza reflexiva y sistemática de la lengua oral.

La muestra está conformada por trece maestros de lengua castellana de educación media pertenecien- 
tes a diez instituciones de carácter público y privado ubicadas en diferentes localidades de la ciudad de Bogotá. Los sujetos seleccionados se caracterizan por su disparidad en cuanto a edad, trayectoria profesional y relación con experiencias de acción pedagógica e investigativa. Se considera que los profesores de lengua castellana como lengua materna son los llamados en primer lugar a comprender y sustentar la importancia de la oralidad como actividad del lenguaje y a proponer acciones didácticas para su desarrollo reflexivo y sistemático. De ahí que este mismo grupo de profesores sean a posteriori los directos beneficiarios y multiplicadores de su impacto.

El estudio de las concepciones sobre la enseñanza de la lengua oral permite responder a las preguntas que guían la investigación: ¿cuáles son las concepciones disciplinares y didácticas de la lengua oral que se manifiestan en el discurso y las prácticas de los profesores de lengua castellana?, ¿qué relaciones se dan y qué implicaciones tienen las concepciones disciplinares y didácticas en las prácticas de enseñanza de la lengua oral en el aula de educación media? y, en general, ¿cuáles son los fundamentos, lineamientos y estrategias derivados de la investigación que sus- tenten una propuesta de formación docente orientada hacia la enseñanza reflexiva y sistemática de la lengua oral en la educación media?

\section{Resultados, conclusiones y propuesta de formación}

Siguiendo las etapas de caracterización, interpretación y reconstrucción de sentido del discurso y la acción didáctica, se logra identificar, por una parte, concepciones didácticas y disciplinares relacionadas con aspectos epistemológicos alrededor del significado otorgado a la oralidad, las condiciones para su enseñanza y los procesos de formación docente en lengua oral, y por otra, se caracterizan concepciones relativas al componente curricular, es decir, aquellas referidas a los objetivos de una didáctica de la oralidad, los contenidos de conocimiento de la lengua oral, las actividades que se proponen, las estrategias que se implementan y las concepciones referidas a la evaluación de los aprendizajes relativos a la lengua oral. A continuación se presentan los resultados relativos a este último componente:

Cuadro 1. Categorías y niveles de progresión de las concepciones disciplinares y didácticas relativas al componente curricular.

\begin{tabular}{|c|c|c|c|c|}
\hline \multicolumn{5}{|c|}{ Metacategoría central: Concepciones disciplinares y didácticas } \\
\hline \multirow{2}{*}{ Categorías } & \multicolumn{4}{|c|}{ Niveles identificados } \\
\hline & \multicolumn{2}{|r|}{ Nivel inicial } & Nivel intermedio & Nivel deseable \\
\hline \multirow{2}{*}{ 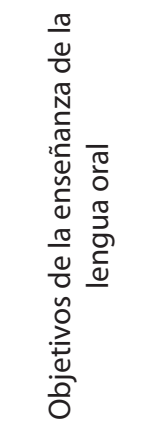 } & 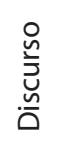 & $\begin{array}{l}\text { N1: La práctica de } \\
\text { actividades orales es- } \\
\text { pontáneas o en pro- } \\
\text { ducción. }\end{array}$ & $\begin{array}{l}\text { N2: Deben considerar aspectos } \\
\text { procedimentales y en menor } \\
\text { medida, aspectos conceptuales } \\
\text { y socioafectivos. }\end{array}$ & $\begin{array}{l}\text { N3: Deben interrelacionar aspectos } \\
\text { procedimentales, conceptuales y } \\
\text { socioafectivos. }\end{array}$ \\
\hline & 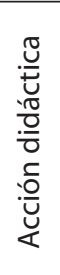 & $\begin{array}{l}\text { N1: El objetivo espe- } \\
\text { cífico consiste en la } \\
\text { comprensión de mo- } \\
\text { delos del habla culta } \\
\text { que los estudiantes } \\
\text { deben apropiar. }\end{array}$ & $\begin{array}{l}\text { N2: El objetivoespecífico consiste } \\
\text { en la instrucción de tópicos } \\
\text { relativos al funcionamiento de } \\
\text { la lengua oral y su puesta en } \\
\text { práctica en actividades que se } \\
\text { simulan o crean eventualmente } \\
\text { en el aula. }\end{array}$ & $\begin{array}{l}\text { N3: El objetivo transversal se orienta al } \\
\text { dominio comprensivo y expresivo de } \\
\text { distintos géneros orales en diversos } \\
\text { contextos, desde un proceso paulatino } \\
\text { que implique una actitud reflexiva sobre } \\
\text { sus mecanismos de uso y sus potenciales } \\
\text { efectos. }\end{array}$ \\
\hline
\end{tabular}




\begin{tabular}{|c|c|c|c|c|}
\hline \multirow{2}{*}{ 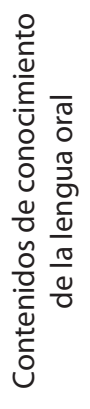 } & 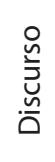 & $\begin{array}{l}\text { N1: Deben orientarse } \\
\text { al dominio de aspec- } \\
\text { tos lingüísticos y nor- } \\
\text { mativos de la lengua. }\end{array}$ & $\begin{array}{l}\text { N2: Se orientan al dominio inme- } \\
\text { diato de sus aspectos comuni- } \\
\text { cativos, contextuales y pragmá- } \\
\text { ticos. }\end{array}$ & $\begin{array}{l}\text { N3: Se secuencian y concretan en } \\
\text { función del dominio paulatino de sus } \\
\text { aspectos contextuales, pragmáticos y } \\
\text { discursivos. }\end{array}$ \\
\hline & 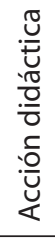 & $\begin{array}{l}\text { N1: Se reemplazan } \\
\text { por actividades orales } \\
\text { ocasionales. }\end{array}$ & $\begin{array}{l}\text { N2: Consideran algunos de } \\
\text { sus aspectos comunicativos } \\
\text { y pragmáticos, que incluyen } \\
\text { conceptos y actitudes, aplicados } \\
\text { a actividades orales ocasionales. }\end{array}$ & $\begin{array}{l}\text { N3: Priman los contenidos manifiestos } \\
\text { en reflexiones sobre los usos sociales de } \\
\text { la lengua oral, sus componentes, las for- } \\
\text { mas de adaptación formal a cada con- } \\
\text { texto e interlocutor y las diferentes ma- } \\
\text { neras de gestionar interacciones orales. }\end{array}$ \\
\hline \multirow[b]{2}{*}{ 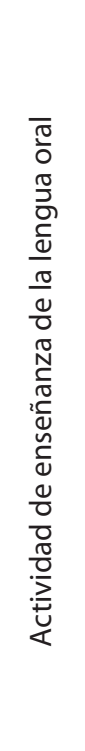 } & 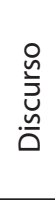 & $\begin{array}{l}\text { N1: No ha sido vincu- } \\
\text { lada a la actividad del } \\
\text { aula. }\end{array}$ & $\begin{array}{l}\text { N2: Ocasionalmente se desarrolla } \\
\text { en el marco de determinadas } \\
\text { situaciones comunicativas. }\end{array}$ & $\begin{array}{l}\text { N3: Permanentemente plantea posibles } \\
\text { hipótesis de trabajo relacionadas con } \\
\text { el dominio comprensivo y expresivo } \\
\text { de distintos géneros orales en diversos } \\
\text { contextos. }\end{array}$ \\
\hline & 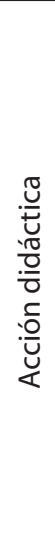 & $\begin{array}{l}\text { N1: El profesor de len- } \\
\text { gua castellana plan- } \\
\text { tea eventualmente } \\
\text { situaciones didácticas } \\
\text { asociadas a temas ar- } \\
\text { ticulados a ejercicios; } \\
\text { algunos, coherentes } \\
\text { con los medios didác- } \\
\text { ticos diseñados en } \\
\text { función de la explica- } \\
\text { ción del profesor rela- } \\
\text { cionada con el uso so- } \\
\text { cial de la lengua oral } \\
\text { en diversos contextos. }\end{array}$ & $\begin{array}{l}\text { N2: El profesor de lengua caste- } \\
\text { llana plantea eventualmente si- } \\
\text { tuaciones didácticas asociadas a } \\
\text { temas articulados a una secuen- } \\
\text { cia de actividades, en su mayo- } \\
\text { ría coherentes con los medios } \\
\text { didácticos diseñados en función } \\
\text { de que los estudiantes tomen } \\
\text { decisiones por sí mismos frente } \\
\text { al uso social de la lengua oral en } \\
\text { diversos contextos. }\end{array}$ & $\begin{array}{l}\text { N3: El profesor de lengua castellana } \\
\text { plantea de manera sistemática situacio- } \\
\text { nes didácticas asociadas a problemas y } \\
\text { contenidos articulados a una secuen- } \\
\text { cia de actividades que, a su vez, son } \\
\text { coherentes con los medios didácticos } \\
\text { diseñados y con las distintas formas de } \\
\text { evaluación que buscan la construcción } \\
\text { de un saber hacer relacionado con el } \\
\text { uso social de la lengua oral en diversos } \\
\text { contextos y niveles de complejidad. }\end{array}$ \\
\hline \multirow{2}{*}{ 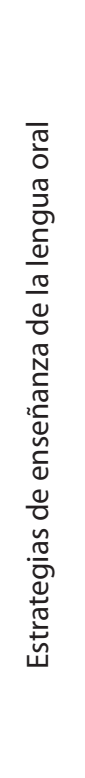 } & 怘 & $\begin{array}{l}\text { N1: En la enseñanza de } \\
\text { la lengua oral se de- } \\
\text { ben contemplar estra- } \\
\text { tegias y técnicas para } \\
\text { desarrollar habilida- } \\
\text { des de memorización. }\end{array}$ & $\begin{array}{l}\text { N2: En la enseñanza ocasio- } \\
\text { nal de la lengua oral se deben } \\
\text { contemplar estrategias para } \\
\text { adquirir y/o desarrollar habili- } \\
\text { dades metacognitivas, proce- } \\
\text { dimentales y socio-afectivas. }\end{array}$ & $\begin{array}{l}\text { N3: En la enseñanza sistemática de la } \\
\text { lengua oral se debe contemplar la in- } \\
\text { terrelación de estrategias metacogniti- } \\
\text { vas, procedimentales y socioafectivas. }\end{array}$ \\
\hline & 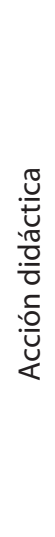 & $\begin{array}{l}\text { N1: El profesor de } \\
\text { lengua castellana de- } \\
\text { muestra dificultades } \\
\text { en la comprensión, } \\
\text { formulación e instru- } \\
\text { mentación de estra- } \\
\text { tegias didácticas y } \\
\text { en su lugar propone } \\
\text { actividades y téc- } \\
\text { nicas para mejorar } \\
\text { la comprensión y/o } \\
\text { producción oral de } \\
\text { los estudiantes en el } \\
\text { contexto académico. }\end{array}$ & $\begin{array}{l}\text { N2: El profesor de lengua cas- } \\
\text { tellana eventualmente diseña } \\
\text { algunas estrategias didácti- } \\
\text { cas encaminadas a mejorar } \\
\text { la comprensión y/o produc- } \\
\text { ción oral de los estudiantes } \\
\text { en el contexto académico. }\end{array}$ & $\begin{array}{l}\text { N3: El profesor de lengua castellana } \\
\text { comprende y diseña de manera siste- } \\
\text { mática estrategias encaminadas a lograr } \\
\text { mayores niveles de apropiación del dis- } \\
\text { curso en diversos contextos, construc- } \\
\text { ción colectiva de los aprendizajes, cua- } \\
\text { lificación de la discusión cooperativa. }\end{array}$ \\
\hline
\end{tabular}




\begin{tabular}{|c|c|c|c|c|}
\hline 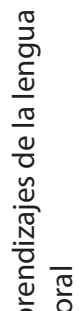 & 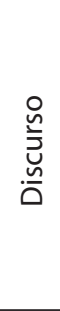 & $\begin{array}{l}\text { N1: La evaluación de } \\
\text { los aprendizajes de } \\
\text { la lengua oral debe } \\
\text { centrarse en la par- } \\
\text { ticipación oral del } \\
\text { estudiante y especíi- } \\
\text { camente, en aspectos } \\
\text { lingüísticos. }\end{array}$ & $\begin{array}{l}\text { N2: La evaluación ocasional de } \\
\text { los aprendizajes de la lengua } \\
\text { oral debe centrarse en la com- } \\
\text { prensión y producción oral y } \\
\text { específicamente, en aspectos } \\
\text { lingüísticos y pragmáticos. }\end{array}$ & $\begin{array}{l}\text { N3: La evaluación sistemática y proce- } \\
\text { sual de los aprendizajes de la lengua } \\
\text { oral debe centrarse en el proceso com- } \\
\text { prensivo y expresivo de distintos géne- } \\
\text { ros orales en diversos contextos y, es- } \\
\text { pecíficamente, en aspectos lingüísticos, } \\
\text { pragmáticos e interactivos. }\end{array}$ \\
\hline 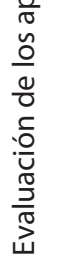 & 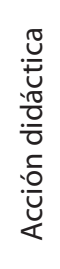 & $\begin{array}{l}\text { N1: La evaluación de } \\
\text { los aprendizajes de la } \\
\text { lengua oral en el nivel } \\
\text { medio de enseñanza } \\
\text { no se realiza. }\end{array}$ & $\begin{array}{l}\text { N2: La evaluación ocasional de } \\
\text { los aprendizajes de la lengua } \\
\text { oral en el nivel medio de ense- } \\
\text { ñanza contempla algunos as- } \\
\text { pectos pragmáticos limitados a } \\
\text { las características del contexto } \\
\text { de uso. }\end{array}$ & $\begin{array}{l}\text { N3: La evaluación sistemática y proce- } \\
\text { sual de los aprendizajes de la lengua } \\
\text { oral en el nivel medio de enseñanza } \\
\text { contempla aspectos pragmalingüísticos } \\
\text { y sociodiscursivos y actúa como estrate- } \\
\text { gia para reflexionar en los mecanismos } \\
\text { de uso oral. }\end{array}$ \\
\hline
\end{tabular}

a) La población docente analizada considera que los objetivos de la enseñanza de la oralidad deben orientarse a la instrucción de tópicos relativos al funcionamiento de la lengua oral y al desarrollo de actividades orales. Subyace, entonces, la intención de abordar aspectos conceptuales, comunicativos y socioafectivos de la oralidad; no obstante, en los objetivos trazados en la acción didáctica estos aspectos no logran interrelacionarse. De ahí que la finalidad dada a la enseñanza de la oralidad por la población docente estudiada se enfoque a la inmediatez de su ejercitación, en detrimento de su poder estructurante. Es decir, se promueve una oralidad espontánea o en producción con unos propósitos comunicativos inmediatos que no contemplan procesos reflexivos sobre sus propios mecanismos de uso.

b) Respecto de los contenidos de conocimiento de la lengua oral, un $29 \%$ de los profesores participantes enfatiza en la necesidad de una orientación formal y normativa; un $50 \%$, en el dominio inmediato de aspectos comunicativos, funcionales y pragmáticos de la oralidad, y un $21 \%$ coinciden en que los principales contenidos de conocimiento de la lengua oral se secuencian y concretan en función del dominio paulatino de sus aspectos contextuales, pragmáticos y discursivos. Se demuestra, entonces, una concepción orientada a superar el reduccionismo normativo y avanzar en la integración de aspectos conceptuales, procedimentales y actitudinales de la lengua oral. Esta tendencia se evidencia parcialmente en la acción didáctica, donde predominan temas y no contenidos curriculares; a pesar de ello, se tocan aspectos comunicativo-pragmáticos y, en menor medida, conceptuales y actitudinales, los cuales de articulan a determinadas actividades orales.

Se advierte de nuevo cierta ausencia de conocimiento del objeto de aprendizaje, no solo en la selección, secuenciación y concreción de contenidos de conocimiento de la lengua oral, sino en su misma construcción y razón de ser y en el grado de dificultad de las actividades orales propuestas. Es decir, se requiere que el maestro cuente con unos criterios que orienten la selección y articulación curricular. Para tal fin, es fundamental partir del reconocimiento de las concepciones previas de los estudiantes, sus necesidades sociales y su propia idiosincrasia, en conjunción con la observación reflexiva de las situaciones orales que se plantean.

c) En relación con la concepción que se tiene de la enseñanza de la lengua oral, a pesar de que un 55\% del profesorado reconoce que no ha la asumido de manera sistemática en el nivel medio de educación o no la ha vinculado a su práctica pedagógica y un 
$35 \%$ aduce que la aborda, pero de manera ocasional, cuando se propone desarrollar una secuencia didáctica sobre lo oral logran plantear situaciones didácticas asociadas a temas articulados a una secuencia de actividades, en su mayoría, coherentes con los medios didácticos, diseñados en función de que los estudiantes tomen decisiones por sí mismos en el uso social de la lengua oral en diversos contextos. En la reflexión sobre esta intervención didáctica, el mismo grupo de docentes coincide en las dificultades que emergieron al intentar articular las situaciones comunicativas orales propuestas al desarrollo de contenidos posibles, como también al diseño de medios didácticos y formas de evaluación.

d) El $16 \%$ del profesorado participante considera que en la enseñanza de la lengua oral se deben contemplar estrategias y técnicas para desarrollar habilidades de memorización. Un $81 \%$, que en la enseñanza ocasional de la lengua oral se deben contemplar estrategias para adquirir y/o desarrollar habilidades metacognitivas, procedimentales y socioafectivas. Y un $3 \%$, que se debe contemplar la interrelación de estrategias metacognitivas, procedimentales y socioafectivas. La concepción de las estrategias didácticas de la oralidad reflejada en la acción didáctica de la mayoría de docentes tiene cierto correlato con estas concepciones previas (en el nivel intermedio y deseable). En la mayoría de los docentes se observó que eventualmente diseñan algunas estrategias didácticas encaminadas a mejorar la comprensión y/o producción oral de los estudiantes en el contexto académico.

e) La evaluación de los aprendizajes relativos a la lengua oral se considera una tarea delicada, en cuanto no se cuenta con criterios claros para su realización y, cuando se ejecuta, esta corresponde a una evaluación sumativa cifrada en la participación oral del estudiante. La ausencia de evaluación de la lengua oral responde, entre otras razones, al desconoci- miento del carácter pedagógico de la oralidad como actividad del lenguaje, a la tradición de limitar la evaluación de la lengua oral a sus aspectos formales o a que se la concibe de naturaleza acústica, efímera, agramatical e informal. Otra razón por la cual en el profesorado de lengua materna se genera cierto rechazo hacia la evaluación del desempeño oral de los estudiantes es la dificultad para realizarla.

En suma, tanto en el discurso como en la acción didáctica se observa que un mismo profesor puede situarse en distintos niveles, derivados de las diferentes hipótesis de progresión, lo cual revela no solo la complejidad de su análisis, sino también la amplia diversidad epistémica y didáctica constitutiva de las concepciones disciplinares y didácticas de los profesores de lengua castellana sobre la oralidad. Por consiguiente, se reconoce el carácter móvil permanente de las concepciones (Figura 1).

Se observa que la mayoría de los profesores avanza de una concepción tradicional (centrada en aspectos normativos de la lengua) acerca de los contenidos de conocimiento, objetivos y evaluación de la lengua oral hacia una concepción comunicativa y contextualista (en que predominan visiones próximas a la enseñanza de la lengua escrita), reflejada especialmente en la implementación de estrategias y actividades orales y en su discurso sobre su importancia y las condiciones para enseñarla, lo cual señala que un mismo profesor posee concepciones distintas de determinados aspectos disciplinares y didácticos de la oralidad. Esta fluctuación puede obedecer a su participación en experiencias innovadoras en otros tópicos de la lengua materna, a su vinculación a redes académicas e investigativas, derivadas de su interés profesional y/o a su formación posgradual o a su iniciativa para realizar trabajos colegiados de transformación de las prácticas docentes en lengua materna. Se trata de condiciones facilitan un mayor nivel de reflexión en y sobre la acción didáctica y, por tanto, 
Figura 1. Nivel de progresión en que se encuentran el conjunto de profesores.

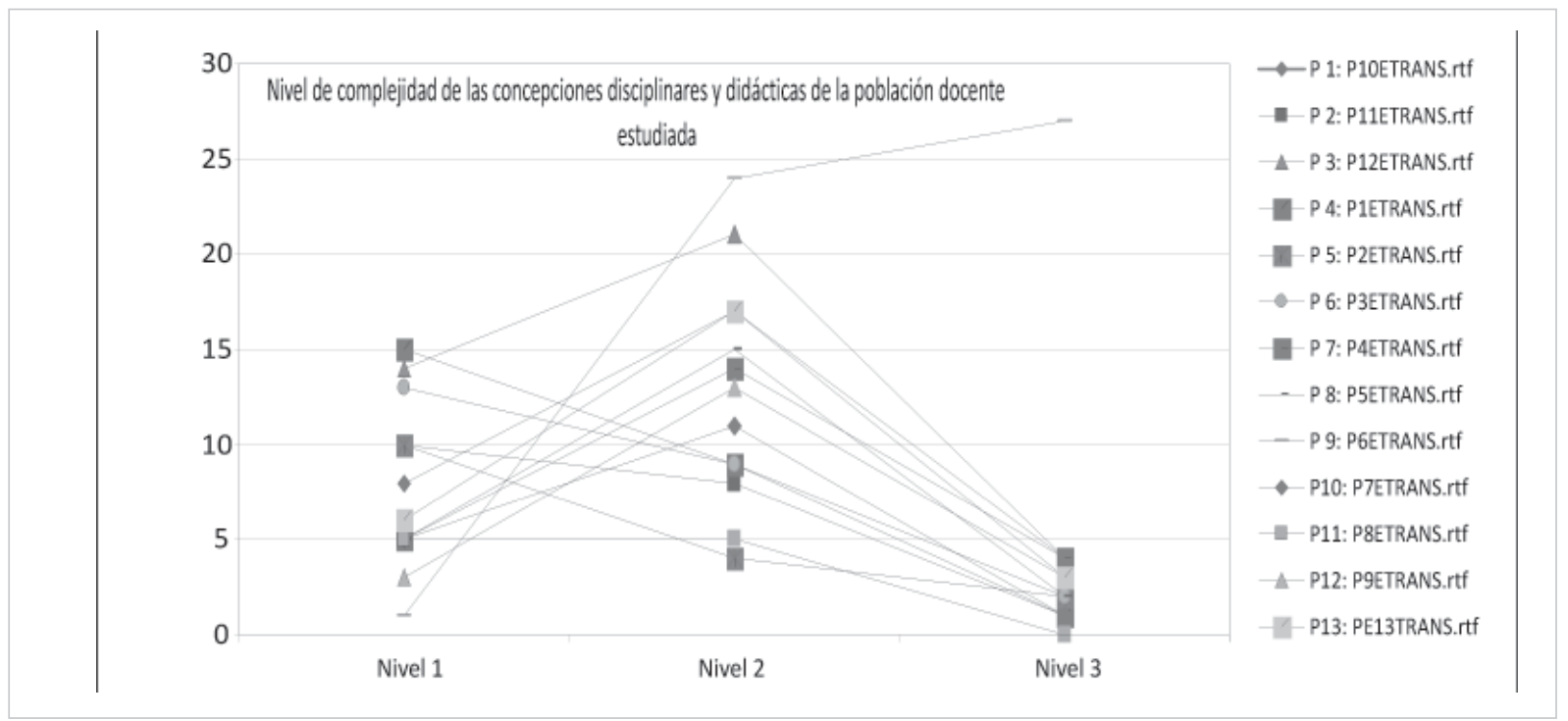

Fuente: elaboración propia

un desarrollo de su propio conocimiento didáctico del contenido.

Por consiguiente, la población docente analizada no representa concepciones únicas, sino una mezcla, a veces con fuertes contradicciones; por tanto, es necesario considerar otros factores que pueden incidir en esta relación entre el decir y el hacer del profesor.

Figura 2. Nivel predominante en las concepciones disciplinares y didácticas de trece profesores de lengua castellana.

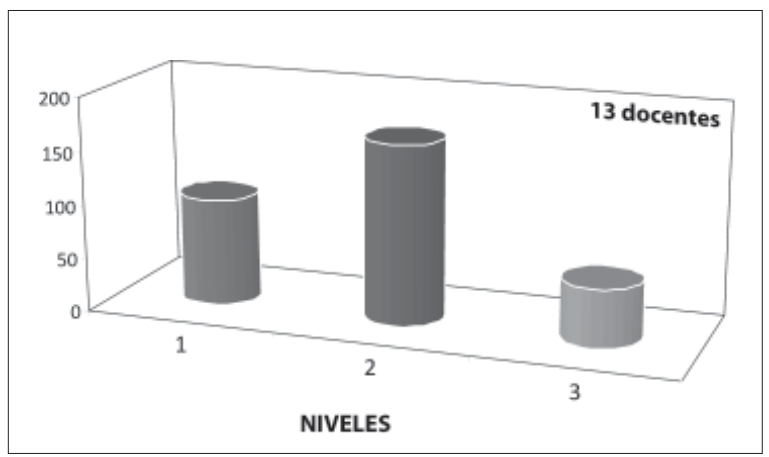

Fuente: Elaboración propia.
Pese a esta complejidad propia del mundo de significados de los profesores, este estudio también da cuenta de una particular forma de matizar la ausencia de dominio pedagógico de un saber relacionado con un tópico de la materia. Los profesores de lengua castellana traslapan hábilmente su inexperiencia y débil dominio de un "conocimiento didáctico del contenido lingüístico-discursivo oral", en un discurso innovador propio de profesores con experiencias transformadoras o que atraviesan por un proceso de cambio en su ejercicio profesional y en una práctica cifrada en cierto activismo centrado en los estudiantes.

En general, se encuentra que los profesores de lengua castellana en su discurso aluden a saberes disciplinares y didácticos relativos a la oralidad, pero no logran integrarlos en su acción didáctica. No obstante, este hallazgo también tiene una lectura positiva, debido a que en su discurso demostraron cierto nivel de conciencia respecto de la oralidad como objeto de enseñanza. Por tanto, construir y/o potenciar el conocimiento didáctico del contenido lingüístico-dis- 
cursivo oral en el grupo de docentes participantes en este estudio es más factible, en razón a que cuentan con una mayor predisposición a reconocer las necesidades e intereses de los estudiantes, en términos de concepciones, y a proponer estrategias variadas, lo cual indica la factibilidad de planificar situaciones didácticas para lograr una interacción más efectiva con el medio didáctico y avanzar hacia la evolución conceptual o la construcción de conocimientos escolares desde enfoques de enseñanza potencialmente efectivos.

En consecuencia, el conocimiento didáctico de la lengua oral configura un dispositivo vital del conocimiento del profesor y resulta decisivo en el desarrollo profesional de los docentes de lengua castellana, en cuanto se forman en esta disciplina específica y construyen un conocimiento especializado o experto del contenido a enseñar, con la pretensión de transformarlo en un saber escolar. De igual forma, en los docentes en ejercicio el "conocimiento didáctico del contenido lingüístico-discursivo oral" es susceptible de potenciación desde una actitud reflexiva del profesor o a través del fortalecimiento de una actividad investigativa permanente cifrada en la reflexión en y sobre la acción didáctica.

Esta necesidad formativa implica al Estado, las facultades de educación y las escuelas normales superiores en la formación de docentes profesionales, con un pensamiento reflexivo, crítico y sistemático sobre su práctica pedagógica y una actitud transformadora de su realidad. Los programas de formación y actualización docente en lenguaje están llamados a favorecer el desarrollo del conocimiento didáctico del contenido lingüístico-discursivo oral, si se pretende que los profesores aborden la enseñanza de la lengua materna de manera integral desde la construcción de sus propios modelos didácticos derivados de esquemas de acción que reflejan una práctica pedagógica reflexiva y sistemática.
Esta pretensión implica la articulación de los distintos actores comprometidos en la formación de formadores, de tal manera que las acciones encaminadas a la construcción del conocimiento didáctico del contenido lingüístico-discursivo oral logren actuar coordinadamente y aporten al propósito de mejoramiento de la enseñanza de la lengua castellana como lengua materna. En esta propuesta convergen fundamentos disciplinares (ontológicos, gnoseológicos, axiológicos, antropológicos, retóricos, lingüísticodiscursivos), pedagógicos y didácticos de la oralidad, en cuanto se reconoce su naturaleza interdisciplinar. Por tanto, se proyecta brindar a los maestros herramientas teóricas y metodológicas que les faciliten la construcción gradual de un conocimiento sistemático y reflexivo sobre la enseñanza y el aprendizaje de la lengua oral.

"Los lineamientos para la autoformación docente en didáctica de la oralidad" son una propuesta que busca favorecer la evolución y el desarrollo de algunas concepciones disciplinares y didácticas de los profesores de lengua castellana, teniendo en cuenta que estas transitan por diferentes niveles en un gradiente de complejidad creciente. Cabe reiterar que la hipótesis de progresión del conocimiento profesional no presenta una trayectoria lineal por la que todos los profesores deban pasar, más bien se trata de una confluencia de ciclos, niveles y fluctuaciones (Imbernón), representados en un espiral de progresiva complejidad que alterna momentos de reflexión, de estudio y de actuación y posibilita que sean los mismos maestros quienes controlen los procesos de enseñanza y aprendizaje, en la perspectiva de construir un modelo didáctico personal (Porlán y Rivero, 1998). La propuesta se organiza a partir de una perspectiva disciplinar, pedagógica y didáctica, expuesta de manera sintética de este modo:

En el ámbito disciplinar, se fundamenta en la perspectiva sociointeraccionista discursiva, una opción 
epistemológica planteada por Bronckart (2004), a fin de ahondar en la problemática de la enseñanza de las lenguas. Específicamente, se asume desde tres ángulos. En primer lugar, se busca iluminar la secuencia de actividades de enseñanza y aprendizaje desde una perspectiva reflexiva, situada $y$, por tanto, mediada por el lenguaje; es decir, reconocer que las actividades verbales pasan por un proceso de planificación, regulación y evaluación. En segundo lugar, reconocer que el uso social de la actividad verbal posibilita la coconstrucción de los participantes, quienes desarrollan y/o se apropian de instrumentos semióticos que les facilitan la reflexión sobre su realidad mediata e inmediata. Finalmente, organizar la actividad verbal situada, reflexiva e intersubjetiva mediante la comprensión de los géneros discursivos y particularmente a través de los géneros orales ${ }^{13}$. En este marco se proponen cuatro dimensiones: contextual, discursiva, cognitiva y metadiscursiva, e interdisciplinar de la lengua oral, las cuales constituyen un marco lingüístico-discursivo e interdisciplinar y concretan su interrelación en distintas prácticas discursivas elaboradas, a fin de fortalecer la competencia discursiva oral de los estudiantes.

En los ámbitos pedagógico y didáctico, los lineamientos se apoyan en las tres perspectivas metadisciplinares fundamentadas en el "modelo didáctico de investigación en la escuela"14 de la red de Investigación y Renovación Escolar (IRES) (Porlán y Rivero, 1998). Se trata de las perspectivas constructivista; sistémica y compleja, y crítica. Son tres perspectivas flexibles y complementarias que responden al come- tido de transformar las concepciones disciplinares y didácticas de la lengua oral, en el sentido de adecuarlas, cualificarlas, mejorarlas o complejizarlas, teniendo un punto de referencia o de progreso que motive a avanzar en el desarrollo del profesiona ${ }^{15}$.

Desde la perspectiva constructivista, se orienta la construcción del conocimiento didáctico del contenido lingüístico-discursivo oral, a partir de las concepciones identificadas (que ponen en juego conocimientos procedentes de fuentes curriculares, experienciales, disciplinares, etc.) y su incidencia en las prácticas de enseñanza de la lengua oral en el aula de educación media. Se pretende reconocer la problemática de enseñanza de este tópico específico de la lengua materna y contrastarla con estudios afines. Lo anterior supone una toma de decisiones reflexionadas acerca de qué actitudes e ideas cambiar y por qué hacerlo; así como el diseño de dispositivos y la incorporación de nuevas estrategias a la acción didáctica. Desde la perspectiva sistémica y compleja, se enfatiza en la reflexión sobre un sistema de conceptos, procedimientos y valores que paulatinamente logran un mayor grado de estructuración y generalización. En este sentido, la perspectiva crítica coadyuva a la configuración de posturas ante el sistema de ideas en construcción y sus posibilidades de complejización, el funcionamiento del currículo y del aula y, en general, los objetos de enseñanza y aprendizaje en cuestión.

A este marco de referencia se articula un dispositivo de autoformación docente, cuya realización se

13 Los géneros discursivos orales se conciben como elementos organizadores de lo oral público y/o de la enseñanza de la oralidad en contextos formales de uso, por cuanto representan el dominio de situaciones de comunicación oral de carácter formal o público (escolares, laborales, etc.) y dan cuenta de una correspondencia mutua entre el uso discursivo y su funcionalidad social.

14 Corresponde a un modelo de enseñanza y aprendizaje constructivista e investigativo que entiende el conocimiento escolar como el resultado de una transposición e integración de conocimientos diversos (Porlán y Rivero, 1998).

15 Pérez Gómez llama a este proceso reconstrucción, sustentado en la idea de concebir como posible un cambio gradual o evolutivo del pensamiento y la actuación de los profesores. 
concreta en tres estrategias y modalidades de autoformación docente en investigación cifradas en los principios de autonomía y desarrollo profesional autodirigido. Son estrategias de autoformación articuladas al desarrollo de procesos investigativos del profesor basados en problemáticas curriculares que emergen de una serie de situaciones problémicas en didáctica de la oralidad adecuadas a cada una de las modalidades de formación. Se trata de situaciones problémicas abiertas y complejas relacionadas con la didáctica de la lengua oral, cuya concreción ocurre a partir de unos planes de actividades que orientan cada modalidad de formación docente, así:

1. Autoformación docente en investigación individualizada ${ }^{16}$. Corresponde a un ciclo inicial y a un proceso de indagación personal y presencial, organizado en cursos o talleres que le proporcionan al profesor instrumentos metodológicos para analizar su saber experiencial y para construir estrategias encaminadas a la resolución de problemas, relevantes de su práctica.

En este proceso es fundamental el acompañamiento de un profesor o asesor que oriente la reflexión en la acción en dos niveles (Camps, Ríos y Cambra). En el primer nivel se presentan experiencias de aula, como juegos de saber que reflejan situaciones que ocurren durante los aprendizajes de la lengua oral. El segundo nivel consiste en el análisis de las maneras como el profesor redefine el juego didáctico o lo reorganiza sobre la marcha ante las eventualidades que se le presentan. Esta reflexión en la acción conlleva identificar y analizar las concepciones del profesor sobre la enseñanza y el aprendizaje de la lengua oral, las cuales se fundamentan en el estudio de las concepciones disciplinares y didácticas de esta investigación.

2. Autoformación docente en investigación gru$\mathrm{pal}^{17}$. Corresponde a un ciclo intermedio próximo al deseable y a un proceso investigativo interpersonal y grupal. El carácter colectivo del trabajo se lleva a cabo mediante la conformación de equipos interdisciplinarios de maestros interesados en el intercambio de experiencias, la formulación de proyectos conjuntos y el desarrollo colectivo del conocimiento profesional.

Se trata de un proceso de reflexión sobre la acción didáctica en los tres niveles propuestos por Camps, Ríos y Cambra. Superados los dos niveles correspondientes a la reflexión durante la acción, el tercer nivel corresponde a la toma de distancia que realiza el profesor entre el momento de la práctica y el de la reflexión, el cual se apoya en reflexiones didácticas sobre el mismo fenómeno. Es un proceso que conlleva un nuevo juego de saber representado en la reorganización de los planes curriculares y acciones didácticas concretas y posibles de ser sistematizadas y analizadas. El cuarto nivel implica la intervención intencionada y sistemática, por cuanto involucra la observación continua, a través de instrumentos que faciliten el proceso de recolección de información, el análisis de los datos obtenidos y su evaluación. En el quinto nivel se revisan las concepciones previas (sistema de ideas y actuaciones profesionales dominantes), a fin de resignificar conceptos, procedimientos y valores implicados iluminados por la lectura crítica de fuentes disciplinares y didácticas.

16 El carácter individualizado del trabajo implica un seguimiento comprometido con unos niveles y ritmos propios y con el tratamiento de problemas curriculares relacionados con la oralidad.

17 Se caracteriza por una autoformación realizada mediante objetos virtuales de aprendizaje y organizada alrededor de problemas, vehiculizados en módulos, de tal forma que el profesor cuente con unas fuentes disciplinares con las cuales esté confrontando permanentemente su saber experiencial. 
3. Autoformación docente en investigación en red $^{18}$. Corresponde a un ciclo de referencia y a un proceso investigativo de grupos y redes que se organizan de manera intergrupal y cooperativa, con el fin de realizar alianzas interinstitucionales y proyectos concertados.

Este ciclo se centra en la reflexión sobre la acción didáctica y la acción sociocultural de colectivos docentes e investigadores de diferentes campos disciplinares en torno a la oralidad. Para tal, fin se parte del intercambio de saberes y experiencias académicas e investigativas, con el propósito de identificar el estado actual y las tendencias de las investigaciones relativas a la oralidad, dentro y fuera del país. De esta forma, se detectan vacíos, intereses comunes, necesidades y potencialidades, a partir de situaciones reales de enseñanza y aprendizaje de la lengua oral, las cuales se constituyen en objeto de investigación colectiva. En concordancia con esta caracterización, se proyectan nuevas acciones investigativas, a fin de mantener activos los vínculos intergrupales e interinstitucionales. La finalidad de esta estrategia de autoformación será entonces, al decir de Carr y Kemmis, desarrollar teorías arraigadas en los problemas y las perspectivas de la práctica educativa, mediante la reflexión crítica.

Las tres modalidades o estrategias de formación presentadas contienen principios y problemas que las orientan y cada ciclo contempla un objetivo central, unos objetivos específicos, una situación problémica y un itinerario de problemas adecuado a cada uno de los niveles que conforman los ciclos. Tal como lo ilustra la Figura 3:

En consecuencia, la autoformación se entiende como un proceso de autoaprendizaje determinado por el
Figura 3. Ciclo inicial o ciclo de formación docente en investigación individualizada.

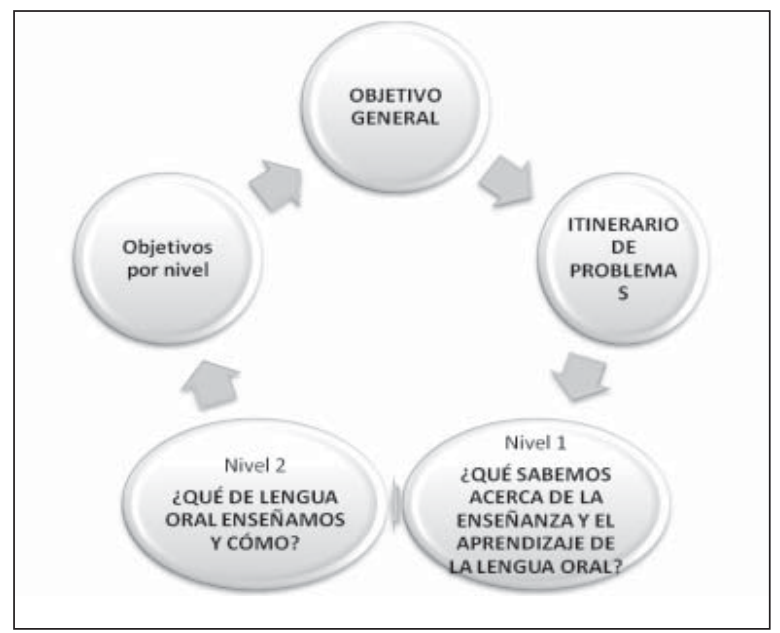

Fuente: Elaboración propia.

ritmo individual, el cual se articula a unos objetivos colectivos. La concreción de este dispositivo implica la realización de principios éticos y didácticos orientados a consolidar una autonomía profesional en el profesor y la evolución del perfil de formador al rol de acompañante.

Cabe reiterar que los lineamientos para la formulación de una propuesta de formación docente son referentes flexibles $y$, por tanto, puntos de partida posibles de ser negociados y modificados de acuerdo con la realidad educativa particular. También se reconoce que el hecho de proponer una situación problémica al profesor no resuelve el problema de transformación de sus concepciones; además, se requiere de un acompañamiento continuo en la elaboración conjunta de herramientas teóricas y metodológicas que le faciliten estructurar su pensamiento y avanzar en el ideal de la colegialidad que favorece las relaciones interdisciplinares y reivindica la profesionalidad docente. Es decir, propender por un educador cons-

18 Se trata de un programa de formación en redes donde el concepto de autoformación docente como desarrollo profesional lleva aparejado el concepto de autonomía en la colegialidad y ésta solo es posible cuando el profesor se vincula a un proyecto común (Imbernón, 2009), coherente con un el ideal de un profesor investigador que participa en proyectos de impacto académico y social. 
ciente de la dimensión institucional, política y social de su práctica pedagógica.

\section{Reconocimientos}

Este artículo es producto de la tesis doctoral "Análisis de las concepciones disciplinares y didácticas sobre la lengua oral en la escuela colombiana actual: un estudio sobre el desarrollo de la competencia discursiva oral en Educación Media”. dirigida por las Dras. María Elvira Rodríguez Luna y Gladys Jaimes de Casadiego. Grupo de investigación Lenguaje, Cultura e Identidad (Categoría A1 de Colciencias)

\section{Bibliografía}

Abascal, M. (2004). La teoría de la oralidad. Universidad de Málaga: Anejo XLIX de Analecta Malacitana.

Bajtín, M. (1982). "El problema de los géneros discursivos". En Estética de la creación verbal (pp. 248-290). México: Siglo XXI.

Brünner, J. J. (1998). Globalización cultural y posmodernidad. Chile: Fondo de Cultura Económica.

Bourdieu, P. (2001). ¿Qué significa hablar? Economía de los intercambios lingüísticos. 3 ed. Madrid: Akal.

Bronckart, J. P. (2004). Actividad verbal, textos y discursos. Por un interaccionismo sociodiscursivo. España: Fundación Infancia y Aprendizaje.

Bronckart, J. P. (1980). Teorías del lenguaje. Introducción crítica. Barcelona: Herder.

Bronckart, J. P. (1985). Las ciencias del lenguaje: ¿Un desafío para la enseñanza? París: Unesco.
Calsamiglia, H. y A. Tusón (1999). Las cosas del decir. Manual de análisis del discurso. Barcelona: Ariel.

Camps, A. (2005). "La lengua oral formal objeto de enseñanza”. En M. Vilá (comp.), El discurso oral formal. Contenidos de aprendizaje y secuencias didácticas (pp. 7-9). España: Graó.

Camps, A. (1993). "Didáctica de la lengua: la emergencia de un campo científico específico". Revista Infancia y Aprendizaje, 62-63, 209-217.

Castoriadis, C. (1997). El avance de la insignificancia. Encrucijadas del Laberinto IV. Buenos Aires: Eudeba.

Clark, C. y P. Peterson (1990). "Procesos de pensamiento de los docentes". En M. Wittrock (comp.), La investigación de la enseñanza III (cap. 6). Barcelona: Paidós.

Contreras, I. (2009). "Vestigios de la oralidad". En I Contreras y M. Collino (coords.), Escritos sobre oralidad (pp. 41-56). México: Universidad Iberoamericana.

Cortés, L. (2002). Los estudios del español hablado entre 1950 y 1999: Períodos, disciplinas y corrientes. Anejos de la revista Oralia: Análisis del discurso oral. Madrid: Arco Libros.

De Certeau, M. (1979). La invención de lo cotidiano. El arte de hacer. T. I. 1 ed. México: Universidad Iberoamericana.

Dijk, T. van (2000). "El discurso como estructura y proceso. Estudios sobre el discurso I". En T. van Dijk (comp.), Una introducción multidisciplinaria. Barcelona: Gedisa.

Dolz, J.; R. Gagnon y S. Mosquera (2009). « La didáctica de las lenguas: una disciplina en proce- 
so de construcción”. Didáctica. Lengua y Literatura, 21, 117-141.

Foucault, M. (1973). El orden del discurso. Barcelona: Túsquets.

Foucault, M. (1978). La voluntad de saber. Historia de la sexualidad. Vol. 1. Madrid: Siglo XXI.

Freire, P. (1969). La educación como práctica de la libertad. Montevideo: Tierra Nueva. [Reedición Siglo XXI].

García, J. J. y P. Cañal (1995). “ ¿Cómo enseñar? Hacia una definición de las estrategias de enseñanza por investigación". Investigación en la Escuela, 25, 5-16.

Halliday, M. A. K. (1989). "Functions of language". En M. A. K. Halliday y R. Hasan (eds.), Language, context, and text: Aspects of language in a social-semiotic perspective (pp. 15-28). Oxford: Oxford University Press.

Jaimes G. y Rodríguez M. E. (2000). “El desarrollo de la oralidad en el preescolar. Práctica cognitiva, discursiva y cultural". Anuario Oralidad, $8,30-38$.

Lomas, C. (2006). "Usos orales en la escuela”. En C. Lomas (comp.), Enseñar lenguaje para aprender a comunicar(se) (pp. 69-73). Bogotá: Editorial Magisterio. Colección Redes.

Lugarini, E. (2006). "Hablar y escuchar. Por una didáctica del 'saber hablar' y del 'saber escuchar"'. Enseñar lenguaje para aprender a comunicar(se) (pp. 69-73). Bogotá: Editorial Magisterio. Colección Redes.

Lyotard, J. (2004). La condición posmoderna: Informe sobre el saber. Madrid: Cátedra.
Marcelo, C. (1987). El pensamiento del profesor. Barcelona: CEAC.

Martínez, M. C. (1991). "La dimensión dialógica del lenguaje". En Analyse du discours des manuels scolaires de sciences ou la sémantique du social et la sémantique de la nature, un écodiscours. Université de Paris XIII.

Núñez, M. P. (2003a). Didáctica de la comunicación oral: Bases teóricas y orientaciones metodológicas para el desarrollo de la competencia discursiva oral en la educación obligatoria. España: Grupo Editorial Universitario.

Núñez, M. P. y A. Romero (2003b). Investigación en didáctica de la lengua e innovación curricular. España: Universidad de Granada.

Núñez, M. P. (2001). Comunicación y expresión oral. Hablar, escuchar y leer en Secundaria. Madrid: MEC-Narcea.

Perafán, G. (2004). La epistemología del profesor sobre su propio conocimiento profesional. Bogotá: Universidad Pedagógica Nacional.

Perafán, G. y A. Adúriz-Bravo (comps.) (2005). Pensamiento y conocimiento de los profesores. Debate y perspectivas internacionales. Bogotá: Editorial Nomos, Universidad Pedagógica Nacional.

Pérez Gómez, Á. y J. Gimeno (1988). “Pensamiento y acción en el profesor: de los estudios sobre la planificación al pensamiento práctico". Infancia y Aprendizaje, 42, 37-63.

Plane, S.yC.García-Debanc(2004). "L'enseignement de 1'oral: enjeux et évolution”. En S. Plane y C. García-Debanc (coords.), Comment enseigner l'oral á l'école primaire? (pp. 7-23). París: Hatier Pedagogie. 
Porlán, R. (1987). "El maestro como investigador en el aula. Investigar para conocer, conocer para enseñar". Investigación en la Escuela, 1, 63-70.

Porlán, R. y P. Cañal (1986). "Una escuela para la Investigación”. Cuadernos de Pedagogía, 134, 45-47.

Porlán, R.; P. Azcárate, R. Martín, J. Martín y A. Rivero (1996). "Conocimiento profesional deseable y profesores innovadores: fundamentos y principios formativos". Investigación en la Escuela, 29, 23-38.

Porlán, R. y A. Rivero (1998). El conocimiento de los profesores. Sevilla: Díada.

Porlán, R.; A. Rivero y R. Martín Del Pozo (1998). "Conocimiento profesional y epistemología de los profesores II: Estudios empíricos y conclusiones". Enseñanza de las Ciencias, 16(2), 271289.

Rey, A.; I, Borja, G. Jaimes y M. E. Rodríguez (en prensa). El lenguaje en Colombia. Aspectos pedagógicos de la lengua. T. II: Historia de la filología y la lingüística en Colombia. Bogotá: Academia Colombiana de la Lengua.

Reyzábal, M. V. (1993). La comunicación oral y su didáctica. Madrid: La Muralla.

Rodríguez J. A. (2000). Posmodernidad, literatura $y$ otras yerbas. Bogotá: Pontificia Universidad Javeriana.
Shulman, L. S. (2001). "Conocimiento y enseñanza. Ensayo”. Estudios Públicos, 83, 163-196.

Shulman, L. S. (1986a). "Those who understand: Knowledge growth in teaching". Educational Researcher, 15(2), 4-14.

Shulman, L.S. (1986b). "Paradigmas y programas de investigación en el estudio de la enseñanza: una perspectiva contemporánea". En M. Wittrock (comp.), La investigación de la enseñanza. I: Enfoques, teorías y métodos. Madrid: Paidós.

Thompson, A. G. (1992). “Teachers' beliefs and conceptions: A synthesis of the research". En D. A. Grouws (ed.), Handbook of research in mathematics teaching and learning (pp. 127-146). Nueva York: Macmillan.

Valbuena, E. (2007). "El conocimiento profesional del profesor de Biología. Estudio de las concepciones de futuros docentes de la Universidad Pedagógica Nacional (Colombia)". Tesis doctoral, Universidad Complutense de Madrid.

Vigotsky, L. S. (1991). Obras escogidas, vol. I. Madrid: Visor.

Vilá, M. (2004). "La actividad oral e intervención didáctica en las aulas". Glosas Didácticas, 12, 113-120.

Winne, P. y Marx, R. (1977). "Reconceptualising research on teaching". Journal of Educational Psychology, 69, 668-678. 